\title{
Emotional Ability and Associative Learning: How Experiencing and Reasoning about Emotions Impacts Evaluative Conditioning
}

\author{
JONATHAN HASFORD \\ BLAIR KIDWELL \\ DAVID M. HARDESTY
}

\begin{abstract}
We extend evaluative conditioning research by examining how differences in emotional ability impact implicit and explicit attitude formation from conditioning. Across five studies, the ability to experience emotional information enhanced the valence of implicit attitudes toward a conditioned stimulus (CS). Conversely, the ability to reason about emotional information reduced the impact of implicit CS attitudes on subsequent explicit evaluations. Furthermore, we examine how brand familiarity and the timing of conditioned and unconditioned stimulus pairings impacts attitude formation. Implications for associative learning and persuasion are provided.
\end{abstract}

Keywords: emotional ability, evaluative conditioning, implicit attitudes, attitude formation

$I^{2}$ ndividuals are frequently the target of persuasion attempts that aim to alter attitudes toward a particular object or idea. A fundamental persuasion tactic is evaluative conditioning (EC; Gibson 2008; Gorn 1982; Olson and Fazio 2001; Sweldens, van Osselaer, and Janiszewski 2010), which involves pairing an unconditioned stimulus (US; e.g., an attractive image) that elicits an automatic response with a conditioned stimulus (CS; e.g., an unknown product or brand). This forms an association between the

Jonathan Hasford (jonathan.hasford@ucf.edu) is an assistant professor of marketing, University of Central Florida, Orlando, FL 32826. Blair Kidwell (blair.kidwell@unt.edu) is an associate professor of marketing, University of North Texas, Denton, TX 76203. David M. Hardesty (david. hardesty@uky.edu) is the Gatton Endowed Chair of Marketing, University of Kentucky, Lexington, KY 40506. Please address correspondence to Jonathan Hasford. The authors thank the JCR review team for their excellent guidance throughout the review process. Supplementary materials are included in the web appendix accompanying the online version of this article.

Editor: Vicki Morwitz

Associate Editor: Stijn van Osselaer

Advance Access publication April 2, 2018
CS and a valenced response (Gorn 1982), which impacts subsequent CS evaluations.

Recent conditioning research has focused on how explicit CS evaluations can differ in valence from implicit associations formed during conditioning. For instance, one can mitigate explicit CS evaluations by instructing individuals to reverse their attitudes toward a CS (Balas and Gawronski 2012; Hütter and Sweldens 2018; Zanon et al. 2014), by activating persuasion knowledge (Gibson, Redker, and Zimmerman 2014; Sweldens et al. 2010), and by increasing cognitive load when individuals are evaluating novel stimuli (Dedonder et al. 2010). These cognitive processing manipulations provide evidence that both associative learning and propositional reasoning processes influence evaluative judgments of a CS (Gawronski and Bodenhausen 2006).

While these studies provide important insights on how individuals may intentionally control their explicit attitudes toward a CS, evaluative conditioning is affective in nature (Dempsey and Mitchell 2010; Sweldens et al. 2010) and involves a transfer of affective properties from the US to the CS (De Houwer, Thomas, and Baeyens 2001; Hofmann et al. 2010). Thus, differences in how people process this emotional information are likely to have a significant 
impact on the valence of attitudes formed after EC. Yet, surprisingly, research has been quite limited on the affective mechanisms underlying the formation and recall of implicit and explicit attitudes from conditioning (De Houwer 2011). Furthermore, Hofmann et al. (2010) concluded, after a meta-analysis of 214 conditioning studies, that future studies need to be shifted away from single-process models of EC and more toward identifying specific affective mechanisms that contribute to the formation of both implicit and explicit attitudes from conditioning. They call for further investigation into new potential moderators that "have consistent effects across different instantiations of EC" and suggest that these moderators may "depend on the type of process that produces a particular EC effect" (416).

We take these insights as a point of departure and investigate how differences in the ability to process emotional information may determine how attitudes are formed from EC. Emotional ability (also known as ability-based emotional intelligence; Kidwell, Hardesty, and Childers 2008a) captures an individual's ability to recognize, process, understand, and manage emotional information in decision making (Mayer, Salovey, and Caruso 2000). We propose that emotional ability plays a critical role in shaping both the associations formed in memory from conditioning, as well as how those associations impact subsequent explicit CS evaluations.

Specifically, we study two fundamental areas of emotional ability-how people experience emotions and how they reason about emotions-to examine how they influence attitude formation processes within EC. Across five studies, we highlight how experiencing emotions enhances the creation of implicit associations from conditioning, while reasoning about emotions reduces the influence of those implicit associations on subsequent explicit CS evaluations. Furthermore, we examine boundary conditions of these effects, including EC with mature versus novel brands and the nature of CS-US pairings (sequential vs. simultaneous). Together, these findings extend EC research by identifying how differences in the way individuals process emotional information can influence both implicit and explicit attitude formation from conditioning.

\section{CONCEPTUAL BACKGROUND}

Several recent studies (Dedonder et al. 2010; Halbeisen et al. 2014; Zanon et al. 2014; Zimmerman, Redker, and Gibson 2011) have identified that both implicit attitudes and explicit deliberation contribute to valenced judgments of a CS. The Associative-Propositional Evaluation model (Gawronski and Bodenhausen 2006) provides a framework for examining how both associative learning and propositional reasoning influence explicit CS evaluations. In conditioning, pairing a CS with one or more USs creates memory links that facilitate the transfer of affect between stimuli. A subsequent association is formed with the CS and the evaluative response, either directly where the CS possesses a firsthand link to valenced nodes or indirectly through associations to the US (Sweldens et al. 2010). An implicit attitude toward the CS emerges that is consistent with the valence of learned associations. This implicit attitude will then influence explicit CS evaluations depending on whether propositional reasoning about the learned associations occurs (Gawronski and Bodenhausen 2006). Propositional reasoning involves the examination and evaluation of associations as an input to subsequent judgments. If propositional reasoning is limited or validates existing associations, then evaluative judgments of a CS will reflect the valence of CS associations. However, if propositional reasoning reduces the influence of CS associations on evaluative judgments, the valence of those judgments should be mitigated.

Multiple factors can influence both the creation of implicit associations from EC as well as the influence of those associations on subsequent explicit evaluations (for a detailed review, see Hofmann et al. 2010). For example, whether CS-US pairings are simultaneous or sequential in nature impacts both the formation of valenced associations in memory, as well as whether explicit CS evaluations are driven through direct consideration of the CS or indirectly through thoughts of the US (Hütter and Sweldens 2013; Sweldens et al. 2010). Furthermore, limitations on individual processing, such as cognitive load, can mitigate the impact of implicit associations on explicit CS evaluations (Dedonder et al. 2010) and lead choice behavior to be based on implicit (vs. explicit) CS attitudes in decisions involving familiar brands (Gibson 2008). However, despite the important role of identifying affective information and relying on valenced affect in judgments after EC, research has not considered how differences in the way people process emotions might influence both associative and propositional processes involved in conditioning.

\section{Emotional Ability}

Emotional ability (EA) reflects an individual's ability to reason about and apply emotional knowledge when achieving a desired outcome (Mayer and Salovey 1997). It encompasses consumers' ability to perceive and interpret emotions, understand how emotions make them feel, and regulate their emotions (Kidwell, Hasford, and Hardesty 2015). EA has been shown to be more important than even IQ in understanding decision making and human behavior (Mayer et al. 2000). Higher EA has widely been linked to a variety of favorable behavioral outcomes (see Martins, Ramalho, and Morin 2010 for a review), including making superior product choices (Kidwell et al. 2008a), relying less on impulsive visual cues in decision making (Kidwell, Hardesty, and Childers 2008b), improving various interpersonal relationships (Caruso and Salovey 2004; Fitness 
FIGURE 1

CONCEPTUAL MODEL OF EMOTIONAL ABILITY AND EVALUATIVE CONDITIONING

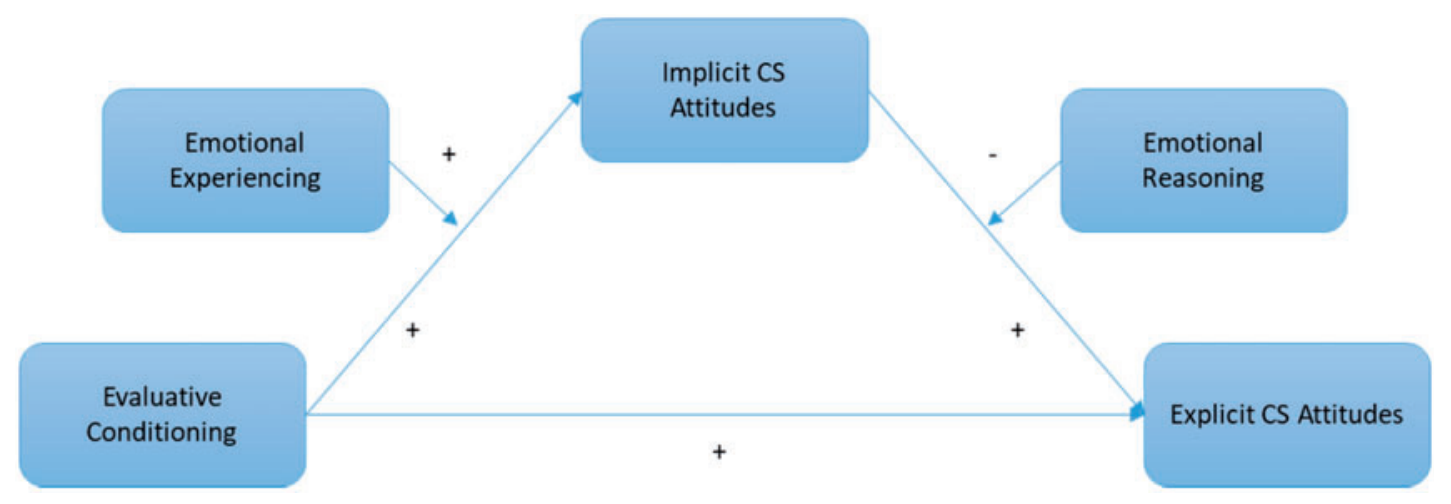

2001; Lopes et al. 2004), and enhancing the quality of marketing exchanges and service interactions (Kidwell et al. 2011; Yim, Tse, and Chan 2008).

The four dimensions of emotional ability can be categorized into two areas described as emotional experiencing (experiential domain: composed of perceiving emotion and facilitating thought) and emotional reasoning (strategic domain: composed of understanding and regulating emotion; Mayer et al. 2000). These areas are associated with different emotional processes that correspond with system $1 /$ system 2 information processing.

Emotional Experiencing. This area of emotional ability reflects the accurate perception, appraisal, and expression of emotions. Experiencing emotions is a relatively intuitive type of information processing that is bound within system 1 processing and is primarily nonconscious and nonverbal in nature (Caruso and Salovey 2004). The ability to experience emotions is also critical in identifying patterns and associations across interrelated sets of information (Brackett, Mayer, and Warner 2004). For example, if an advertisement is attempting to persuade consumers to buy a new brand of beer, the ad may feature smiling models that automatically elicit a favorable affective response. As one's ability to experience emotions increases, these nonconscious emotional responses may be stronger as a result of one's ability to more readily associate the feelings with the product and more vividly relate consumption to the desired affective state.

Emotional Reasoning. This area of emotional ability reflects a mental capacity to analyze emotions and understand their outcomes. Reasoning about emotions involves the more deliberative system 2 processing toward managing emotions in the context of individual goals, selfknowledge, and social awareness (Averill and Nunley 1992; Gross 1998; Ortony, Clore, and Collins 1988;
Roseman 1984). Individuals with enhanced emotional reasoning abilities integrate affective responses with more reasoned cognitions prior to making explicit judgments (Ortony et al. 1988). Consider the previous example. As their ability to reason about emotions increases, individuals become more likely to consider the validity and diagnosticity of the emotional associations created from the advertisement. These individuals may more readily recognize that these associations were designed to impact their behavior, and, as a result, become more likely to control their explicit evaluations of the beer brand.

We propose that both the experiential and reasoning dimensions of emotional ability influence both the associative and propositional reasoning processes that occur during EC. Our conceptual model is provided in figure 1. Specifically, we predict that a greater ability to experience emotions should enhance the creation of implicit attitudes from conditioning. Experiential emotional ability is related to the strength of one's nonconscious affective responses (Caruso and Salovey 2004), and individuals with a greater ability to experience emotions more automatically and intuitively link affective responses to their original source (Brackett et al. 2004). In the context of conditioning, this entails associating the CS with the valenced response elicited by the US. More effectively recognizing and appraising one's feelings should enhance the associative link established by CS-US co-occurrences. As a result, we expect that as their ability to experience emotions increases, individuals should possess stronger implicit CS attitudes consistent with the valence of conditioning.

We also predict that the ability to reason about emotional information will impact attitude formation processes from EC. Because emotional reasoning occurs through slower, more deliberative processes (Averill and Nunley 1992; Roseman 1984) and individuals with enhanced reasoning abilities integrate their feelings with more reasoned 
cognitions (Ortony et al. 1988), we expect that heightened reasoning abilities will reduce the impact of implicit CSUS associations on subsequent explicit CS evaluations. A greater ability to reason about emotions has been linked to a reduced reliance on affective cues in product choice (Kidwell et al. 2008b). In extending this finding to EC, we predict that when individuals possess a greater ability to reason about emotion information, they will more carefully analyze and consider the CS-US associations established from conditioning. These individuals should be more likely to recognize that the US merely provides a cue for CS evaluations and more readily recognize that these affective cues were designed to impact their attitudes. As a result, these individuals should be more likely to disregard the CS-US associations established from conditioning and subsequent explicit CS evaluations should become more neutral in valence.

\section{Plan of Studies}

To examine how emotional ability impacts EC, we conducted five studies. In study 1 , we examine how the experiential and reasoning areas of emotional ability moderate EC effects. Then, in study 2 we replicate our findings by examining EC involving the negative conditioning of brands. In study 3 , we further support our conceptual model by using an EC procedure that features both positive and negative USs. Lastly, studies 4 and 5 test the robustness of our conceptual model by manipulating brand familiarity and the timing of CS-US pairings.

\section{STUDY 1}

To begin, we examine whether the experiential and reasoning areas of emotional ability are associated with EC effects. We expect that the ability to experience emotions will enhance the formation of implicit CS attitudes from conditioning. Conversely, we expect the ability to reason about emotions to reduce the effect of implicit CS attitudes on explicit CS evaluations.

\section{Method}

Two hundred twenty-three undergraduate students were randomly assigned to a single factor (perceptual similarity: high or low) between-subjects design. Participants began by completing an EC procedure adapted from recent research (Sweldens et al. 2010). In this procedure, participants viewed a slide show of various images. The slide show included six fictitious beer brands to serve as CSs. In the low perceptual similarity condition, the slide show also featured three favorable images (a child smiling, wild flowers in bloom, and a forest scene) and three neutral images (a battery pack, an office chair, and a person reading the news) to serve as USs. Furthermore, six additional neutral images (e.g., an airplane, an electrical plug) were included to disguise the nature of the conditioning procedure.

The high perceptual similarity condition featured identical brands and images except for the favorable USs used in conditioning. The positive images (adults smiling at a bar, people walking on the beach, and adults enjoying beer at a cookout) were chosen based on their perceptual similarity to beer. While De Houwer et al. (2001) notes that perceptual similarity between the CS and US can generate demand artifacts in EC, we included a high perceptual similarity condition for two reasons. First, many marketers use perceptually similar stimuli in their communications; thus, doing so here allows us to enhance the external validity of our findings. Secondly, we can also test whether any observed EC effects can be attributed to affect-asinformation processes. According to an affect-asinformation explanation, incidental feelings are more likely to enter evaluations of a target if there is a perceived relatedness between the feelings and the target object (Pham 1998). Since a high level of perceptual similarity signals that the US is related to the CS, we tested whether our conceptual model was limited to EC involving perceptually similar stimuli. If our moderating effects are observed only with perceptually similar stimuli, this would suggest that these effects likely rest on higher-order inferential processes similar to those of affect-as-information. However, if these moderating effects are observed with both perceptually similar and dissimilar stimuli, this would suggest that processes other than affect-as-information drive these effects.

At the beginning of the procedure, a cover story stated that the study was designed to assess college students' attitudes toward beer brands. Participants were also told that it was unlikely that they had seen the brands before, so they would view a slide show to familiarize them with the different brands (Sweldens et al. 2010). Participants then viewed the slide show.

The conditioning procedure consisted of five rounds of sequential CS-US pairings. In each round, one of the positive or neutral USs appeared prior to a beer brand. Each brand was randomly assigned to appear after the same US across the five rounds. Furthermore, the six additional neutral images were interspersed between CS-US pairings. All stimuli appeared on the center of the screen for 1.5 seconds each, with a 0 second interstimulus interval. The order of CS-US pairings and neutral images was completely randomized within each round. In total, each beer brand was paired with the same positive or neutral CS five times across the conditioning procedure. All stimuli and procedural details are available in the web appendix.

After the conditioning procedure, we assessed explicit attitudes toward the beer brands. Participants rated each of the six beer brands across four attitude measures (bad/ good, negative/positive, unsatisfactory/satisfactory, and dislike/like on seven-point bipolar scales; average per 
brand $\alpha=.96$ ). The average attitudes toward the three positively conditioned beer brands and the three neutral brands were computed as measures of explicit CS attitudes.

Next, participants completed manipulation checks. Participants rated each US on a nine-point bipolar scale (extremely negative/extremely positive). These ratings served as a manipulation check for the valence of each US that appeared in the slide show. Participants also rated each US on a five-point scale (not at all related to beer/extremely related to beer) assessing the perceptual similarity of the US to the CS product category. The ratings were averaged for the three positive USs and served as a manipulation check of perceptual similarity.

Participants then completed the Affective Misattribution Procedure (AMP; Payne et al. 2005) to measure implicit CS attitudes. In the AMP, participants are instructed to make a series of fast judgments. In the task, participants first view a prime for 75 milliseconds, followed by a blank screen for 125 milliseconds, and then a Chinese pictograph for 100 milliseconds. After the pictograph, participants see a static visual display and are asked to evaluate the pictograph as either favorable or unfavorable. The static display remains until the participant responds.

In the AMP, the primes represent the implicit attitude of interest. To measure implicit CS attitudes, we included each of the six beer brands as primes. Each beer brand appeared six times prior to a pictograph for a total of 36 randomly ordered trials. Following Payne et al. (2005), we computed the number of favorable pictograph evaluations involving the positively conditioned brand primes and the number of favorable pictograph evaluations involving the neutral brand primes as measures of implicit CS attitudes.

Furthermore, to reduce any demand effects in the AMP, we also included specific instructions from Payne et al. (2005). Participants were told that the primes served merely as a warning for the upcoming pictograph and that the names should not impact their judgments of the ideographs. All AMP stimuli and procedural details are provided in the web appendix.

Participants then completed the Consumer Emotional Intelligence Scale (CEIS; Kidwell et al. 2008a) to measure emotional ability. The CEIS is an 18-item measure designed to objectively assess an individual's ability to perceive, facilitate, understand, and manage emotional information. Each item is scored on a 0-1 scale based on expert consensus, which is designed to reduce biases associated with self-report measures of emotional ability (Kidwell et al. 2008a). Since the experiential area of emotional ability includes the ability to perceive and use emotions to facilitate thought, and the reasoning area of emotional ability includes the ability to understand and manage emotions (Mayer et al. 2000), CEIS scores from the four abilities were used to form scores for our two focal areas of interest. Specifically, we summed scores on the nine items from the perceiving and facilitating dimensions to measure emotional experiencing and the nine items from the understanding and managing dimensions to measure emotional reasoning. All CEIS items and scoring details are provided in the web appendix.

\section{Results}

Correlation matrices and complete statistics for all study variables are provided in the web appendix. In study 1 , emotional experiencing and emotional reasoning were not correlated $(r=-.04, p=.55)$. However, in all subsequent studies, emotional experiencing and emotional reasoning were positively correlated ( $r$ s from .24 to .42 , all $p s<.004)$.

Manipulation Checks. To begin, we examined the valence of the USs used in our conditioning procedures. A repeated-measures ANOVA was conducted with average attitudes toward the three positive USs and average attitudes toward the three neutral USs as within-subjects factors and perceptual similarity (high vs. low) as a betweensubjects factor. Results revealed a significant withinsubjects effect, such that participants rated the positive USs more favorably $(M=7.22, \mathrm{SD}=1.53)$ than the neutral USs $(M=4.66, \mathrm{SD}=1.22 ; F(1,221)=464.4, p<.001)$. Furthermore, the between-subjects similarity effect $(F(1$, $221)=.10, p=.75)$ and the interaction of similarity and US attitudes $(F(1,221)=.10, p=.75)$ were nonsignificant.

We also examined the perceptual similarity of the USs used in our conditioning procedures. A $t$-test was used to compare perceptions of the relatedness of the three positive USs to beer. Results revealed that participants in the high similarity condition viewed the USs as more related to beer $(M=3.35, \mathrm{SD}=.70)$ than participants in the low similarity condition $(M=1.58, \mathrm{SD}=.77, t(221)=18.01, p<.001)$.

Brand Attitudes. Next, we examined whether our EC procedures impacted explicit attitudes toward the conditioned brands. A repeated-measures ANOVA was conducted with average attitudes toward the three positively conditioned brands and average attitudes toward the three neutral brands as within-subjects factors and perceptual similarity as a between-subjects factor. Results revealed a significant within-subjects effect, such that participants rated the beer brands paired with positive USs more favorably $(M=4.24, \mathrm{SD}=1.20)$ than the beer brands paired with neutral USs $(M=4.04, \mathrm{SD}=1.11 ; F(1,221)=8.01$, $p=.005)$. Furthermore, the between-subjects similarity effect $(F(1,221)=.40, p=.53)$ and the interaction of similarity and brand attitudes $(F(1,203)=.11, p=.74)$ were nonsignificant.

We also examined whether EC impacted implicit attitudes toward the conditioned brands. A repeated-measures ANOVA was conducted with the total number of favorable pictograph evaluations for images that were preceded by positively conditioned brands and the total number of 
favorable pictograph evaluations for images that were preceded by neutral brands as within-subjects factors and perceptual similarity as a between-subjects factor. Results revealed a significant within-subjects effect, as participants rated the ideographs more favorably when they were preceded by positively conditioned brands $(M=11.36$, $\mathrm{SD}=5.01)$ relative to when they were preceded by neutral brands $\quad(M=10.49, \quad \mathrm{SD}=4.56 ; \quad F(1, \quad 221)=12.03$, $p=.001)$. Furthermore, the between-subjects similarity effect $(F(1,221)=.16, p=.69)$ and the interaction of similarity and brand attitudes $(F(1,221)=1.03, p=.31)$ were nonsignificant.

Tests of Conceptual Model. Recently, the MEMORE macro for SPSS was developed to test mediation effects within-subjects for repeated-measures mediators and DVs (Montoya and Hayes 2017). However, the MEMORE macro is not capable of testing moderation effects between the IV-mediator relationship or moderation effects between the mediator-DV relationship as predicted by our conceptual model. Therefore, a custom macro was developed by Montoya (2017) to examine moderated mediation effects within-subjects. This macro is based on PROCESS model 21 (Hayes 2013), which includes a moderator that impacts the IV-mediator relationship and a second moderator that impacts the mediator-DV relationship.

Using our custom macro, we first examined our predictions with participants in the high perceptual similarity condition. Full results for all studies are available in the web appendix. The index of moderated moderated mediation was significant (Effect $=-.0469,95 \%$ CI [-.0986, -.0093]). Specifically, emotional experiencing was positively related to within-subject differences in implicit attitudes $($ Effect $=.8021, t=2.33, p=.022)$. This finding suggests that when emotional experiencing increases, the implicit EC effect is strengthened as differences in implicit attitudes between the positively conditioned brand and neutral brand are increased. Additionally, emotional reasoning moderated the relationship between implicit CS attitudes and explicit CS evaluations (Effect $=-.0584, t=-2.84, p=.005)$. Furthermore, the index of conditional moderated mediation for emotional reasoning was significant at high (+1 SD) levels of emotional reasoning, but not at low ( $-1 \mathrm{SD})$ and mean levels of emotional reasoning $\left(\mathrm{High}_{\text {Reasoning }}\right.$ effect $=-.0829,95 \%$ CI $[-.1583,-.0237]$, Mean $_{\text {Reasoning }}$ effect $=-.0360,95 \%$ CI $[-.0864, .0022]$, Low $_{\text {Reasoning }}$ effect $=.0109,95 \%$ CI $[-.0456, .0718]$. This suggests that when emotional reasoning increases, the effect of implicit CS attitudes on explicit CS evaluations is significantly reduced. Together, these findings provide initial support for our conceptual model.

Next, we examined our conceptual model with participants in the low perceptual similarity condition. The index of moderated moderated mediation was significant $($ Effect $=-.0551,95 \%$ CI $[-.1371,-.0030])$. Specifically, emotional experiencing was positively related to withinsubject differences in implicit attitudes (Effect $=.8972$, $t=2.54, p=.013$ ). This finding suggests that when emotional experiencing increases, the implicit EC effect is strengthened as differences in implicit attitudes between the positively conditioned brand and neutral brand are increased. Additionally, emotional reasoning moderated the relationship between implicit CS attitudes and explicit CS evaluations $\quad($ Effect $=-.0614, \quad t=-2.27, \quad p=.025)$. Furthermore, the index of conditional moderated mediation for emotional reasoning was significant at mean and high (+1 SD) levels of emotional reasoning, but not at low $\left(-1\right.$ SD) levels of emotional reasoning $\left(\mathrm{High}_{\text {Reasoning }}\right.$ effect $=-.1243,95 \%$ CI $[-.2742,-.0199]$, Mean Reasoning effect $=-.0692,95 \%$ CI $[-.1610,-.0107]$, Low Reasoning effect $=-.0141,95 \%$ CI $[-.0977, .0519]$. This suggests that when emotional reasoning increases, the effect of implicit CS attitudes on explicit CS evaluations is significantly reduced. Together, these findings suggest that our conceptual model is robust to differences in perceptual similarity.

Finally, since our results were not affected by differences in perceptual similarity, we analyzed both conditions simultaneously within our custom macro. Results are displayed in figure 2, which provides further support for our conceptual model.

\section{Discussion}

Study 1 provides initial evidence for our model of emotional ability and evaluative conditioning. Specifically, we demonstrated that the ability to experience emotional information moderated the relationship between EC and implicit CS attitudes, while the ability to reason about emotional information moderated the implicit-explicit CS attitude link. Furthermore, our findings were robust to the perceptual similarity of the USs used in conditioning. This suggests that emotional ability effects in EC are distinct from affect-as-information processes in attitude formation. Next, we examine our conceptual model with EC involving negatively valenced USs.

\section{STUDY 2}

Study 1 used an EC procedure where positively valenced brands were compared to neutral brands within our conceptual framework. However, EC may also involve pairing brands with unfavorable stimuli to negatively impact brand attitudes toward competitors or to reduce the use of harmful products such as tobacco. Thus, to generalize our findings, study 2 used the same procedure but paired some brands with negative images to further explore the moderating role of emotional experiencing and emotional reasoning in EC. 
FIGURE 2

STUDY 1 RESULTS-ALL PARTICIPANTS

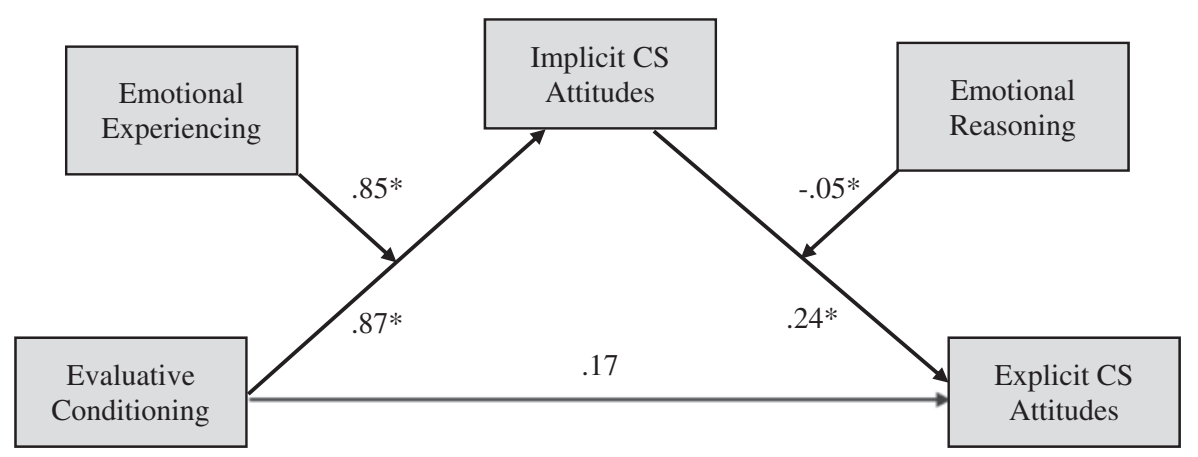

NOTE._All coefficients reported are unstandardized effects. ${ }^{*} p<.001$

\section{Method}

Two hundred forty-two undergraduates were randomly assigned to a single-factor (perceptual similarity: high or low) between-subjects design. Participants completed a conditioning procedure identical to study 1 with one notable difference. In this study, three beer brands were randomly preceded by either a perceptually similar negative US (e.g., images of people getting sick/passed out from drinking alcohol) or a perceptually dissimilar negative US (e.g., a dumpster on fire, a sad dog in a kennel, a rash). All negative USs used in study 2 are available in the web appendix. As a result, three randomly selected beer brands were negatively conditioned, while three randomly selected beer brands were paired with neutral stimuli.

After the conditioning procedure, participants evaluated the brands across the attitude items used in study 1 . The average attitudes toward the three negatively conditioned brands and the average attitudes toward three neutral brands were computed to serve as our measures of explicit CS attitudes. Participants then provided their attitudes toward each US used in conditioning and evaluated the perceptual similarity of all USs to beer using the same items from study 1 . Next, participants completed the AMP procedure to measure implicit attitudes. Then participants completed the CEIS to measure the focal areas of emotional ability. To conclude, participants provided their age and gender and were thanked for their participation.

\section{Results}

Manipulation Checks. To begin, we examined the valence of the USs used in our conditioning procedures. A repeated-measures ANOVA was conducted with average attitudes toward the three negative USs and average attitudes toward the three neutral USs as within-subjects factors and perceptual similarity (high vs. low) as a between-subjects factor. Results revealed a significant within-subjects effect of US attitudes $(F(1,240)=588.3$, $p<.001)$. This main effect was qualified by a significant interaction of US attitudes and perceptual similarity $(F(1$, $240)=6.89, p=.009)$. However, US attitudes followed a similar pattern across perceptual similarity conditions. For participants in the high similarity condition, participants rated the negative USs more unfavorably $(M=2.23$, $\mathrm{SD}=1.51)$ than the neutral USs $(M=5.33, \mathrm{SD}=.96 ; F(1$, $119)=311.2, p<.001)$. For participants in the low similarity condition, participants also rated the negative USs more unfavorably $(M=2.50, \mathrm{SD}=1.45)$ than the neutral USs $(M=4.99, \mathrm{SD}=.91 ; F(1,121)=277.2, p<.001)$.

We also examined the perceptual similarity of the USs used in our conditioning procedures. A $t$-test was used to compare perceptions of the relatedness of the three negative USs to beer. Results revealed that participants in the high similarity condition viewed the USs as more related to beer $(M=4.32, \mathrm{SD}=.76)$ than participants in the low similarity condition $(M=1.65, \mathrm{SD}=.94, t(240)=24.26$, $p<.001)$.

Brand Attitudes. Next, we examined whether our EC procedures impacted explicit attitudes toward the conditioned brands. A repeated-measures ANOVA was conducted with average attitudes toward the three negatively conditioned brands and average attitudes toward the three neutral brands as within-subjects factors and perceptual similarity as a between-subjects factor. Results revealed a significant within-subjects effect, such that participants rated the beer brands paired with negative USs more unfavorably $(M=3.54, \mathrm{SD}=1.28)$ than the beer brands paired with neutral USs $(M=3.76, \mathrm{SD}=1.24 ; F(1,240)=15.51$, $p<.001)$. Furthermore, the interaction of similarity and brand attitudes $(F(1,240)=2.25, \quad p=.14) \quad$ was nonsignificant, while the between-subjects similarity effect 
FIGURE 3

STUDY 2 RESULTS-ALL PARTICIPANTS

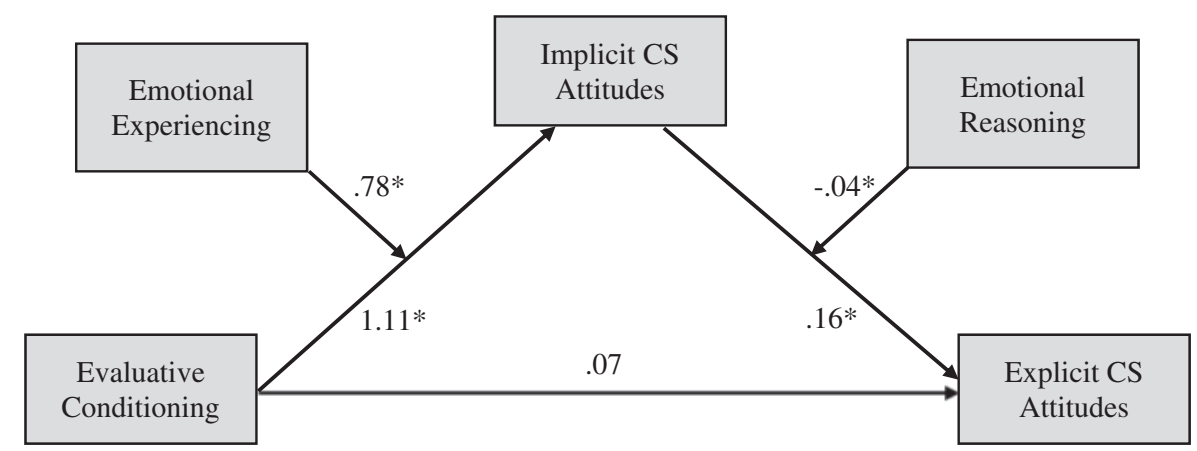

NOTE.-All coefficients reported are unstandardized effects. ${ }^{*} p<.001$

$(F(1,240)=5.44, p=.021)$ was significant. Average attitudes toward all beer brands were lower in the high perceptual similarity condition $(M=3.48)$ relative to the low perceptual similarity condition $(M=3.83)$.

We also examined whether EC affected implicit attitudes toward the conditioned brands. A repeated-measures ANOVA was conducted with the total number of favorable pictograph evaluations for images that were preceded by negatively conditioned brands and the total number of favorable pictograph evaluations for images that were preceded by neutral brands as within-subjects factors and perceptual similarity as a between-subjects factor. Results revealed a significant within-subjects effect, such that participants rated the ideographs less favorably when they were preceded by negatively conditioned brands $(M=10.93, \mathrm{SD}=5.18)$ relative to when they were preceded by neutral brands $(M=12.04, \mathrm{SD}=4.88 ; \quad F(1$, $240)=23.01, p<.001)$. Furthermore, the between-subjects similarity effect $(F(1,240)=.78, p=.38)$ and the interaction of similarity and brand attitudes $(F(1,240)=.14$, $p=.71$ ) were nonsignificant.

Tests of Conceptual Model. Using our custom macro, we tested our conceptual model for participants in the high and low perceptual similarity conditions. For consistency and ease of interpretability, we treated neutral CS attitudes as factor 1 and CS- attitudes as factor 2 in our empirical tests). Thus, the implicit and explicit EC effect should once again be positive, as neutral CS attitudes are more positive in valence relative to explicit CS- attitudes. Furthermore, emotional experiencing should enhance the EC-implicit attitude relationship, while emotional reasoning should reduce the implicit-explicit CS relationship.

In testing our conceptual model, we found that results were once again similar across both the high and low perceptual similarity conditions. Due to space considerations, results by condition are excluded here. Full results are available in the web appendix.

Since our results were not affected by differences in perceptual similarity, we analyzed both conditions simultaneously within our custom macro. Results are displayed in figure 3. The index of moderated moderated mediation was significant (Effect $=-.0302$, 95\% CI [-.0590, -.0073]). Specifically, emotional experiencing was positively related to within-subject differences in implicit attitudes $($ Effect $=.7776, t=3.42, p<.001)$. This finding suggests that when emotional experiencing increases, the implicit EC effect is strengthened as differences in implicit attitudes between the neutral brand and negatively conditioned brand are increased. Additionally, emotional reasoning moderated the relationship between implicit CS attitudes and explicit CS evaluations (Effect $=-.0388, t=-3.72$, $p<.001)$. Furthermore, the index of conditional moderated mediation for emotional reasoning was significant at mean and high $(+1 \mathrm{SD})$ levels of emotional reasoning, but not at low (-1 SD) levels of emotional reasoning $\left(\right.$ High $_{\text {Reasoning }}$ effect $=-.0733,95 \%$ CI $[-.1290,-.0220]$, Mean Reasoning $_{\text {Ren }}$ effect $=-.0432,95 \%$ CI $[-.0795,-.0129]$, Low Reasoning effect $=-.0131,95 \%$ CI $[-.0429, .0116]$. This suggests that when emotional reasoning increases, the effect of implicit CS attitudes on explicit CS evaluations is significantly reduced. Together, these findings provide additional support for our conceptual model.

\section{Discussion}

Study 2 provides further support for our model of emotional ability and evaluative conditioning. Using a procedure designed to negatively condition brands, we provided further evidence of the moderating role of emotional ability in EC. Specifically, the ability to experience emotions led to a greater implicit EC effect, while the ability to reason 
about emotions reduced the impact of implicit brand attitudes on explicit brand evaluations. Next, we examine our conceptual model with an EC procedure designed to positively condition some brands and negatively condition others.

\section{STUDY 3}

Studies 1 and 2 provide initial evidence of the moderating role of emotional ability in EC. In both studies, we produced an EC effect by pairing some brands with valenced USs and other brands with neutral USs. However, EC can also be used to change attitudes by pairing some brands with positively valenced USs and other brands with negatively valenced USs within the same conditioning procedure (De Houwer et al. 2001; Zimmerman et al. 2011). Thus, study 3 further generalizes our conceptual model by examining whether emotional experiencing and emotional reasoning moderate $\mathrm{EC}$ effects when the $\mathrm{EC}$ procedure uses both positively valenced and negatively valenced USs to change consumer attitudes.

\section{Method}

One hundred fifty participants $(41.5 \%$ female, $M_{\text {age }}=37.1$ ) from Amazon Mechanical Turk (MTurk) completed this study for monetary compensation. Participants began by completing a conditioning procedure similar to studies 1 and 2, with one notable difference. In this study, the beer brands were randomly preceded by either a positive US from the low perceptual similarity condition in study 1 or a negative US from the low perceptual similarity condition in study 2 . Thus, in study 3 , three beer brands were positively conditioned and three beer brands were negatively conditioned.

After the conditioning procedure, participants evaluated the brands across the attitude items used in the previous studies. The average attitudes toward each of the three positively conditioned brands and the average attitudes toward each of the three negatively conditioned brands were computed to serve as our measures of explicit CS attitudes. Participants then provided their attitudes toward each US used in conditioning and evaluated the perceptual similarity of all USs to beer using the same items from prior studies. Next, participants completed the AMP procedure from previous studies to measure implicit attitudes. Then participants completed the CEIS to measure the focal areas of emotional ability. To conclude, participants provided their age and gender and were thanked for their participation.

\section{Results}

Two participants did not complete all study measures. Thus, data from 148 participants were usable for analysis.
Manipulation Checks. To begin, we examined the valence of the USs used in our conditioning procedures. A repeated-measures ANOVA was conducted with average attitudes toward the three positive USs and average attitudes toward the three negative USs as a within-subjects factor. Results revealed a significant within-subjects effect, such that participants rated the positive USs more favorably $(M=7.90, \quad \mathrm{SD}=.98)$ than the negative USs $(M=2.00, \mathrm{SD}=.96 ; F(1,147)=2000.6, p<.001)$.

Next, we verified that the USs used in our conditioning procedure were perceptually dissimilar to beer. Onesample $t$-tests were used to compare perceptions of the relatedness of the three positive USs and three negative USs to the midpoint of the relatedness scale (3). Results revealed that participants rated the positive USs significantly below the midpoint of the scale $(M=1.63$, $\mathrm{SD}=.87 ; t(147)=-19.14, p<.001)$. Furthermore, participants also rated the negative USs significantly below the midpoint of the scale $(M=1.20, \mathrm{SD}=.51 ; t(147)=-43.35$, $p<.001)$.

Brand Attitudes. Next, we examined whether our EC procedures impacted explicit attitudes toward the conditioned brands. A repeated-measures ANOVA was conducted with average attitudes toward the positively conditioned brands and average attitudes toward the negatively conditioned brands as a within-subjects factor. Results revealed a significant within-subjects effect, such that participants rated the beer brands paired with positive USs more favorably $(M=4.54, \mathrm{SD}=1.14)$ than the beer brands paired with negative USs $(M=4.17, \mathrm{SD}=1.08$; $F(1,147)=9.34, p=.003)$.

We also examined whether our EC procedures impacted implicit attitudes toward the conditioned brands. A repeated-measures ANOVA was conducted with the total number of favorable pictograph evaluations for images that were preceded by positively conditioned brands and the total number of favorable pictograph evaluations for images that were preceded by negatively conditioned brands as a within-subjects factor. Results revealed a significant within-subjects effect, such that participants rated the ideographs more favorably when they were preceded by positively conditioned brands $(M=11.05, \mathrm{SD}=3.58)$ relative to when they were preceded by negatively conditioned brands $(M=10.12, \mathrm{SD}=3.70 ; F(1,147)=9.82, p=.002)$.

Test of Conceptual Model. Using our custom macro, we obtained results replicating our previous studies. The index of moderated moderated mediation was significant (Effect $=-.0441,95 \%$ CI $[-.0938,-.0038])$. Specifically, emotional experiencing was positively related to withinsubject differences in implicit attitudes $($ Effect $=.6100$, $t=2.07, p=.041)$. This finding suggests that when emotional experiencing increases, the implicit EC effect is strengthened as differences in implicit attitudes between 
the positively conditioned brand and negatively conditioned brand are increased. Additionally, emotional reasoning moderated the relationship between implicit CS attitudes and explicit CS evaluations (Effect $=-.0723$, $t=-2.82, p=.006$ ). Furthermore, the index of conditional moderated mediation for emotional reasoning was significant at mean and high $(+1 \mathrm{SD})$ levels of emotional reasoning, but not at low ( $-1 \mathrm{SD})$ levels of emotional reasoning $\left(\right.$ High $_{\text {Reasoning }}$ effect $=-.1116,95 \%$ CI $[-.1999,-.0340]$, Mean $_{\text {Reasoning }}$ effect $=-.0674,95 \%$ CI $[-.1303,-.0175]$, Low $_{\text {Reasoning }}$ effect $=-.0233,95 \%$ CI $[-.0904, .0314]$. This suggests that when emotional reasoning increases, the effect of implicit CS attitudes on explicit CS evaluations is significantly reduced. Together, these findings provide further support for our conceptual model.

\section{Discussion}

Study 3 provides further support for our conceptual model. Using an EC procedure where some brands were positively conditioned while other brands were negatively conditioned, we obtained results replicating studies 1 and 2. Next, we test the robustness of our conceptual model by investigating EC involving mature brands.

\section{STUDY 4}

Studies 1-3 provide support for our conceptual model of emotional ability and EC using novel brands as CSs. In study 4, we further tested our conceptual model of emotional ability and evaluative conditioning by examining EC with mature brands. Whereas EC is often effective at changing attitudes toward novel brands, previous research suggests that EC may be less successful in altering explicit attitudes toward mature brands (Cacioppo et al. 1992; Shimp, Stuart, and Engle 1991). However, we propose that our conceptual model is robust to differences in brand familiarity.

Gibson (2008) extended research on EC and mature brands by examining both implicit and explicit attitude change for mature brands. Results revealed that while EC did not directly affect explicit brand attitudes, EC did significantly impact implicit attitudes toward mature brands. Furthermore, implicit attitudes were significantly correlated with explicit brand attitudes after conditioning (Gibson 2008, 182) and changes in implicit attitudes subsequently affected explicit choice preferences (Gibson 2008, 184). These findings suggest that EC can affect explicit brand attitudes indirectly through changes in implicit attitudes for mature brands. Because our conceptual model (figure 1) proposes that the dimensions of emotional ability impact the indirect pathways of explicit attitude formation from EC, we predict that our focal dimensions of emotional ability will continue to moderate the attitude formation process from EC with mature brands in a similar manner as EC with novel brands.

\section{Method}

One thousand ninety-two undergraduates were initially given a screening survey at the beginning of the semester. Following procedures from Gibson (2008), we had participants answer explicit preference items toward various soft drink brands, including Coke and Pepsi. The items included seven bipolar attitude items on seven-point scales (bad/good, poor quality/high quality, dislike very much/ like very much, inferior/superior, unattractive/attractive, unpleasant/pleasant, boring/interesting, unfavorable/favorable) and an overall seven-point global evaluation item (Overall, my feeling about [soft drink brand] is unfavorable/favorable). The items for Coke and Pepsi were summed and a difference score (Coke minus Pepsi) was computed. Across the entire sample, a moderate preference for Coke was observed $(M=1.21, \mathrm{SD}=1.91)$. Because Gibson (2008) suggests that implicit attitudes toward mature brands can be affected by EC only if participants have relatively neutral existing preferences, participants for our main study were recruited only if they possessed relatively neutral preferences (within $+/-.5 \mathrm{SD}$ from neutral preference (0) difference score; Gibson 2008) between the two brands.

Two hundred sixty-six participants agreed to participate for course credit. Participants were randomly assigned to a 2 (brand familiarity: fictitious/mature) $\times 2$ (counterbalancing: brand A positive, brand B positive) between-subjects design.

All conditioning procedures were identical to the low perceptual similarity condition in study 1 with the exception of two primary differences. First, instead of pairing three brands with positive images and three brands with neutral images, we paired the three positive images with one focal brand and the three neutral images with the other brand. In the fictitious brand condition, we included two fictitious cola brands (Spartan Cola and Level One Cola). In the mature brand condition, we used Coke and Pepsi as our focal brands (Gibson 2008). Secondly, our stimuli were counterbalanced between-subjects in this study. In one condition, the first cola brand (Spartan for fictitious, Coke for mature) was paired with the positive images while the second cola brand (Level One Cola for fictitious, Pepsi for mature) was paired with neutral images. In the other counterbalancing condition, the second cola brand was paired with positive images while the first cola brand was paired with neutral images. All other procedural details were identical to study 1 . All stimuli used in study 4 are available in the web appendix.

After the conditioning procedure, participants evaluated the brands featured in the conditioning procedure across the attitude items used in previous studies. Then, by 
adapting the items used in prior studies, we had participants provide their attitudes toward each US used in conditioning and evaluate the perceptual similarity of all USs to cola. Next, participants completed the AMP procedure from previous studies to measure implicit attitudes. The AMP was slightly adjusted, such that the two brands featured in the conditioning procedure appeared 18 times each as primes. This number is equivalent to the total number of positive and neutral primes in study 1, and all other procedural details are identical to previous studies. Then participants completed the CEIS to measure the focal areas of emotional ability. To conclude, participants were thanked for their participation.

\section{Results}

Manipulation Checks. To begin, we examined the valence of the USs used in our conditioning procedures. A repeated-measures ANOVA was conducted with average attitudes toward the three positive USs and average attitudes toward the three neutral USs as within-subjects factors and brand familiarity and our conditioning procedure as between-subjects factors. Results revealed a significant within-subjects effect, such that participants rated the positive USs more favorably $(M=6.88, \mathrm{SD}=1.44)$ than the neutral USs $(M=4.65, \quad \mathrm{SD}=.95 ; \quad F(1,262)=484.7$, $p<.001)$. All other main effects and interactions were nonsignificant (all $p \mathrm{~s}>.17$ ).

Next, we examined the perceptual similarity of the USs used in our conditioning procedure. A one-sample $t$-test was used to compare perceptions of the relatedness of the three positive USs to the midpoint of the relatedness scale (3). Results revealed that participants rated the USs significantly below the midpoint of the scale $(M=1.79$, $\mathrm{SD}=.80 ; t(265)=-24.86, p<.001)$, suggesting that the USs were not perceptually similar to the cola brands featured in the conditioning procedure. Additionally, perceptions of relatedness were not affected by our betweensubjects factors or their interaction (all $p \mathrm{~s}>.23$ ).

Brand Attitudes. Next, we examined whether our EC procedures impacted explicit attitudes toward the conditioned brands. A repeated-measures ANOVA with explicit attitudes as a within-subjects factor and brand familiarity and counterbalancing as between-subjects factors was assessed. The within-subjects effect of explicit attitudes $(F(1,262)=3.90, p=.049)$ and the between-subjects effect of brand familiarity $(F(1,262)=11.42, p=.001)$ were significant. All other main effects and interactions were nonsignificant (all $p \mathrm{~s}>.12$ ). For participants in the fictitious brand condition, attitudes toward the positively conditioned brand $(M=3.94, \mathrm{SD}=1.46)$ were more favorable relative to attitudes toward the neutral brand $(M=3.74$, $\mathrm{SD}=1.38 ; F(1,130)=4.65, p=.033)$. For participants in the mature brand condition, however, attitudes toward the positively conditioned brand $(M=4.49, \mathrm{SD}=1.75)$ were not significantly different relative to attitudes toward the neutral brand $(M=4.47, \mathrm{SD}=1.79 ; \quad F(1,132)=.15$, $p=.70)$. This is consistent with previous research (Cacioppo et al. 1992; Gibson 2008; Shimp et al. 1991) suggesting that EC does not meaningfully affect explicit attitudes toward mature brands.

We then examined whether our EC procedures impacted implicit attitudes toward the conditioned brands. A repeated-measures ANOVA with implicit attitudes as a within-subjects factor and brand familiarity and counterbalancing as between-subjects factors was assessed. The within-subjects effect of implicit attitudes $(F(1$, $262)=63.82, p<.001)$ was significant, while the betweensubjects effect of brand familiarity $(F(1,262)=3.73$, $p=.055$ ) was marginally significant. All other main effects and interactions were nonsignificant (all $p s>.47$ ). For participants in the fictitious brand condition, a significant within-subjects effect was observed, as participants rated the ideographs more favorably when they were preceded by the positively conditioned brand $(M=10.01$, $\mathrm{SD}=4.07)$ relative to when they were preceded by the neutral brand $(M=8.08, \mathrm{SD}=4.59 ; F(1,130)=35.46$, $p<.001)$. For participants in the mature brand condition, a significant within-subjects effect was also observed, as participants rated the ideographs more favorably when they were preceded by the positively conditioned brand $(M=10.86, \mathrm{SD}=4.74)$ relative to when they were preceded by the neutral brand $(M=9.25, \mathrm{SD}=5.04 ; F(1$, $132)=28.33, p<.001)$. This finding is consistent with Gibson (2008), who suggests that implicit attitudes toward mature brands can be affected by EC.

Tests of Conceptual Model. Using our custom macro, first we examined our conceptual model with participants in the novel brand condition. The index of moderated moderated mediation was significant $($ Effect $=-.0322,95 \%$ CI $[-.0701,-.0029])$. Specifically, emotional experiencing was positively related to within-subject differences in implicit attitudes $($ Effect $=.7699, t=2.43, p=.017)$. This finding suggests that when emotional experiencing increases, the implicit EC effect is strengthened as differences in implicit attitudes between the positively conditioned brand and neutral brand are increased. Additionally, emotional reasoning moderated the relationship between implicit CS attitudes and explicit CS evaluations (Effect $=-.0418, t=-2.11, p=.037$ ). Furthermore, the index of conditional moderated mediation for emotional reasoning was significant at high $(+1 \mathrm{SD})$, mean, and low $(-1$ SD) levels of emotional reasoning, with the effect size increasing as emotional reasoning increased $\left(\mathrm{High}_{\text {Reasoning }}\right.$ effect $=-.1127,95 \%$ CI $[-.2207,-.0136]$, Mean $_{\text {Reasoning }}$ effect $=-.0805,95 \%$ CI $[-.1654,-.0093]$, Low $_{\text {Reasoning }}$ effect $=-.0483,95 \%$ CI $[-.1206,-.0020]$. This suggests that when emotional reasoning increases, the 
effect of implicit CS attitudes on explicit CS evaluations is reduced. Together, these findings provide further support for our conceptual model.

Next, we examined our conceptual model with participants in the mature brand condition. The index of moderated moderated mediation was significant $($ Effect $=-.0235,95 \%$ CI $[-.0638,-.0005])$. Specifically, emotional experiencing was positively related to withinsubject differences in implicit attitudes $($ Effect $=.8150$, $t=2.77, p=.006)$. This finding suggests that when emotional experiencing increases, the implicit EC effect is strengthened as differences in implicit attitudes between the positively conditioned brand and neutral brand are increased. Additionally, emotional reasoning moderated the relationship between implicit CS attitudes and explicit CS evaluations $\quad($ Effect $=-.0288, \quad t=-2.29, \quad p=.024)$. Furthermore, the index of conditional moderated mediation for emotional reasoning was significant at high $(+1 \mathrm{SD})$ and mean levels of emotional reasoning, but not at low ( $-1 \mathrm{SD})$ levels of emotional reasoning $\left(\mathrm{High}_{\text {Reasoning }}\right.$ effect $=-.0697$, 95\% CI $[-.1627,-.0028]$, Mean Reasoning $_{\text {effect }}=-.0462$, 95\% CI [-.1080, -.0018], Low Reasoning $_{\text {effect }}=-.0227,95 \%$ CI $[-.0661, .0026]$. This suggests that when emotional reasoning increases, the effect of implicit CS attitudes on explicit CS evaluations is significantly reduced. Together, these findings suggest that our conceptual model is robust to differences in CS familiarity.

\section{Discussion}

Study 4 demonstrates the robustness of our conceptual model of emotional ability and evaluative conditioning by examining EC with mature brands. In an EC procedure involving mature brands (Coke and Pepsi), explicit attitudes were indirectly affected by changes in implicit attitudes post-conditioning. Furthermore, our focal dimensions of emotional ability once again moderated both the formation of implicit attitudes from EC as well as the impact of implicit attitudes on explicit judgments of conditioned brands. Next, in study 5, we examine how the timing of CS-US pairings impacts our conceptual model.

\section{STUDY 5}

While studies 1-4 provide support for our conceptual model of emotional ability and evaluative conditioning, each study used a sequential conditioning procedure where the US preceded the CS. However, EC can also involve simultaneous CS-US pairings to elicit attitude change (Hofmann et al. 2010). Therefore, study 5 extends our conceptual model by examining how emotional ability moderates conditioning effects when the CS and US are presented simultaneously during the conditioning procedure.
When the CS and US are presented simultaneously, implicit misattribution underlies attitude change (Sweldens et al. 2010). Implicit misattribution involves the misattribution of US attitudes to the CS outside of conscious awareness, which can produce a lasting effect on CS attitudes (Hütter and Sweldens 2013; Jones et al. 2010). Importantly, implicit misattribution is characterized as a type of intrinsic learning in which attitude formation is not dependent on the recognition of CS-US associations (Sweldens, Corneille, and Yzerbyt 2014, 200). Confusability of the original source of one's feelings occurs with simultaneous CS-US pairings, as individuals are unsure of which co-occurring stimuli in their environment are producing a valenced response (Hütter and Sweldens 2013). Because emotional experiencing increases the valence of affective responses by enhancing awareness of the original source of one's feelings (Brackett et al. 2004), this dimension of emotional ability should not impact attitude formation processes when individuals are unsure of where their feelings initially originate. As a result, we predict that in an EC procedure involving simultaneous CS-US pairings, the ability to experience emotions will no longer moderate the formation of implicit attitudes from EC. However, we continue to expect that emotional reasoning will reduce the relationship between implicit and explicit attitudes.

\section{Method}

Two hundred five participants $(41.5 \%$ female, $\left.M_{\text {age }}=37.1\right)$ from MTurk were randomly assigned to a single-factor (conditioning: sequential/simultaneous) between-subjects design. In the sequential condition, participants completed the EC procedure from study 1 with USs that were low in perceptual similarity to beer brands. In the simultaneous condition, the same stimuli were used and procedures were adapted from Sweldens et al. (2010). All images during the conditioning phase appeared in pairs onscreen. The USs and conditioned brands appeared next to each other onscreen and were positionally counterbalanced (left vs. right side of screen) across trials. The pairs appeared for 3 seconds each, with a 0 second interval between stimuli. Furthermore, all neutral stimuli were shown in pairs onscreen for 3 seconds each as well and were also positionally counterbalanced across trials. This procedure guaranteed an equal slide show duration and total presentation duration of CS-US pairs across conditions.

After the conditioning procedure, participants evaluated the conditioned and neutral brands across the attitude items used in the previous studies. The average attitudes toward each of the three positively conditioned brands and the average attitudes toward each of the three neutral brands were computed to serve as our measures of explicit CS attitudes. Then, using the same items from study 1, we had participants provide their attitudes toward each US used in 
conditioning and evaluate the perceptual similarity of all USs to beer. Next, participants completed the AMP procedure from study 1 to measure implicit attitudes. Then participants completed the CEIS to measure the focal areas of emotional ability. To conclude, participants provided their age and gender and were thanked for their participation.

\section{Results}

Manipulation Checks. To begin, we examined the valence of the USs used in conditioning. A repeatedmeasures ANOVA was conducted with average attitudes toward the three positive USs and average attitudes toward the three neutral USs as within-subjects factors and our EC procedure (sequential vs. simultaneous) as a betweensubjects factor. Results revealed a significant withinsubjects effect, such that participants rated the positive USs more favorably $(M=7.46, \mathrm{SD}=1.40)$ than the neutral USs $(M=5.04, \mathrm{SD}=1.09 ; F(1,203)=514.7, p<.001)$. Furthermore, the between-subjects conditioning factor $(F(1,203)=.75, p=.39)$ and the interaction of conditioning and US attitudes $(F(1,203)=.40, p=.53)$ were nonsignificant.

Next, we examined the perceptual similarity of the USs used in our conditioning procedures. A one-sample $t$-test was used to compare perceptions of the relatedness of the three positive USs to the midpoint of the relatedness scale (3). Results revealed that participants rated the USs significantly below the midpoint of the scale $(M=1.54$, $\mathrm{SD}=.81 ; t(204)=-25.83, p<.001)$, suggesting that the USs were not perceptually similar to the beer brands featured in the conditioning procedure. Additionally, perceptions of relatedness did not differ between the sequential and simultaneous conditions $(t(203)=-.88, p=.38)$.

Brand Attitudes. Next, we examined whether our EC procedures impacted explicit attitudes toward the conditioned brands. A repeated-measures ANOVA was conducted with average attitudes toward the three positively conditioned brands and average attitudes toward the three neutral brands as within-subjects factors and our conditioning procedure (sequential vs. simultaneous) as a betweensubjects factor. Results revealed a significant withinsubjects effect, as participants rated the beer brands paired with positive USs more favorably $(M=4.74, \mathrm{SD}=1.20)$ than the beer brands paired with neutral USs $(M=4.45$, $\mathrm{SD}=1.07 ; F(1,203)=13.36, p<.001)$. Furthermore, the between-subjects conditioning factor was nonsignificant $(F(1,203)=.13, p=.72)$, while the interaction of conditioning and brand attitudes $(F(1,203)=3.79, p=.053)$ was marginally significant.

We also examined whether our EC procedures impacted implicit attitudes toward the conditioned brands. A repeated-measures ANOVA was conducted with the total number of favorable pictograph evaluations for images that were preceded by positively conditioned brands and the total number of favorable pictograph evaluations for images that were preceded by neutral brands as within-subjects factors and our conditioning procedure (sequential vs. simultaneous) as a between-subjects factor. A significant interaction of conditioning and implicit attitudes was observed $(F(1,203)=5.06, p=.026)$. However, implicit attitudes followed a similar pattern across conditions. For participants in the sequential condition, participants rated the ideographs more favorably when they were preceded by positively conditioned brands $(M=11.80, \mathrm{SD}=4.85)$ relative to when they were preceded by neutral brands $(M=8.80, \mathrm{SD}=6.05 ; F(1,102)=19.53, p<.001)$. For participants in the simultaneous condition, participants also rated the ideographs more favorably when they were preceded by positively conditioned brands $(M=11.49$, $\mathrm{SD}=3.84)$ relative to when they were preceded by neutral brands $\quad(M=10.20, \quad \mathrm{SD}=4.42 ; \quad F(1, \quad 101)=15.04$, $p<.001)$.

Tests of Conceptual Model. Using our custom macro, first we examined our conceptual model with participants in the sequential condition. The index of moderated moderated mediation was significant $($ Effect $=-.0857,95 \% \mathrm{CI}$ $[-.2038,-.0071])$. Specifically, emotional experiencing was positively related to within-subject differences in implicit attitudes (Effect $=1.6648, t=2.50, p=.014)$. This finding suggests that when emotional experiencing increases, the implicit EC effect is strengthened as differences in implicit attitudes between the positively conditioned brand and neutral brand are increased. Additionally, emotional reasoning moderated the relationship between implicit CS attitudes and explicit CS evaluations (Effect $=-$ $.0515, t=-2.68, p=.009)$. Furthermore, the index of conditional moderated mediation for emotional reasoning was significant at high (+1 SD) and mean levels of emotional reasoning, but not at low ( -1 SD) levels of emotional reasoning $\left(\mathrm{High}_{\text {Reasoning }}\right.$ effect $=-.2400,95 \%$ CI $[-.4892,-.0463]$, Mean $_{\text {Reasoning }}$ effect $=-.1544$, 95\% CI $[-.3142,-.0314]$, Low $_{\text {Reasoning }}$ effect $=-.0689,95 \%$ CI $[-.1953, .0164]$. This suggests that when emotional reasoning increases, the effect of implicit CS attitudes on explicit CS evaluations is significantly reduced. Together, these findings further support our conceptual model.

We then examined the moderating role of emotional ability in attitude formation for participants who completed the simultaneous conditioning procedure. Using our custom macro based on PROCESS model 21, results differed in the simultaneous condition. Specifically, the index of moderated moderated mediation was not significant (Effect $=.0020,95 \%$ CI $-.0453, .0479]$ ) and emotional experiencing did not impact differences in implicit brand attitudes (Effect $=-.0323, t=-.10, p=.92$ ). These findings suggest that differences in emotional experiencing do 
FIGURE 4

STUDY 5 RESULTS—SIMULTANEOUS CS-US PAIRINGS

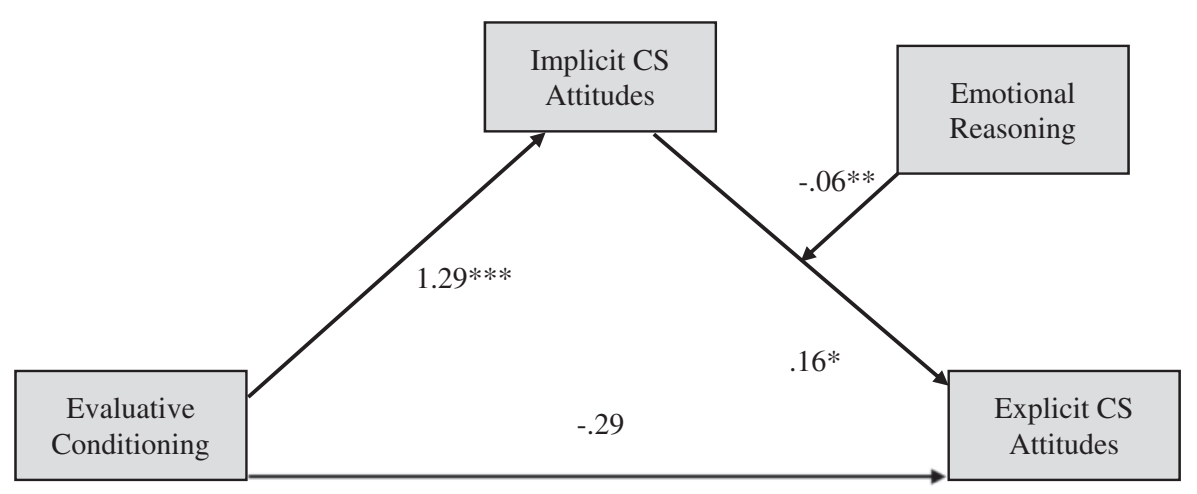

NOTE.-All coefficients reported are unstandardized effects. ${ }^{*} p<.05 .{ }^{* *} p<.01 .{ }^{* * *} p<.001$

not moderate the effect of EC on implicit attitude formation.

Thus, to examine how emotional ability affects differences in attitude formation from simultaneous EC pairings, we used a custom macro developed by Montoya (2017) for testing mediated moderation effects (see Hayes 2013, PROCESS model 14) within-subjects. Results (see figure 4) supported our predictions regarding EC with simultaneous pairings. Specifically, a significant within-subjects effect of EC on implicit brand attitudes was observed $($ Effect $=1.294, \quad t=3.88, \quad p<.001)$. Additionally, emotional reasoning moderated the relationship between implicit CS attitudes and explicit CS evaluations (Effect $=-.0610, t=-2.77, p=.007)$. Furthermore, conditional indirect effects for emotional reasoning were significant at high $(+1 \mathrm{SD})$ levels of emotional reasoning, but not at mean and low ( -1 SD) levels of emotional reasoning $\left(\right.$ High $_{\text {Reasoning }}$ effect $=-.1451,95 \%$ CI [-.2850, -.0325], Mean $_{\text {Reasoning }}$ effect $=-.0300,95 \%$ CI [-.1087, .0604], Low $_{\text {Reasoning }}$ effect $=.0852,95 \%$ CI $[-.0366, .2778]$. This suggests that when emotional reasoning increases, the effect of implicit CS attitudes on explicit CS evaluations is significantly reduced. Together, these findings support our predictions regarding simultaneous EC and the moderating role of emotional reasoning in explicit attitude formation.

\section{Discussion}

Study 5 identifies how emotional ability impacts conditioning effects when the timing of CS-US pairings is manipulated. For sequential CS-US pairings, results replicated our previous studies. However, when the conditioned brands were simultaneously paired with valenced USs, the ability to experience emotions no longer moderated the formation of implicit attitudes from conditioning. Together, these findings highlight a boundary condition to our conceptual model of emotional ability and EC.

\section{GENERAL DISCUSSION}

The current research extends the evaluative conditioning literature by examining how two fundamental areas of emotional ability impact EC effects. Specifically, the ability to experience emotions facilitated the creation of valenced implicit CS attitudes. Conversely, the ability to reason about emotions reduced the impact of implicit CS attitudes on subsequent explicit evaluations. This conceptual model was robust to differences in perceptual similarity of the USs used in conditioning and brand familiarity. Furthermore, we identified that when EC involves simultaneous CS-US pairings, emotional experiencing no longer impacted the formation of implicit CS attitudes. These findings provide both theoretical and practical implications.

\section{Theoretical Contributions}

A number of emerging studies (Balas and Gawronski 2012; Dedonder et al. 2010; Gibson et al. 2014; Hütter and Sweldens 2013; Zanon et al. 2014) have identified various cognitive processing manipulations that can impact explicit attitude formation from EC. Our research extends this literature by examining how affective processing mechanisms influence both implicit and explicit CS attitudes. Because EC involves the transfer of affect between stimuli (Hofmann et al. 2010), examining differences in how individuals recognize, use, understand, and manage feelings and emotional responses is critical to understanding how EC impacts attitude formation processes. However, prior research has been limited in considering how EC effects 
are influenced by differences in an individual's ability to process affective information. We address this gap by highlighting how two fundamental areas of emotional ability (emotional experiencing and emotional reasoning) impact both implicit and explicit attitude formation after EC.

Our research also provides implications for general learning and information processing. While evaluative conditioning is a fundamental type of associative learning (Olson and Fazio 2001; Sweldens et al. 2010), we expect that emotional ability should be impactful in any type of learning that involves the co-occurrence of signals and outcomes. For example, consider a traditional behavioral learning paradigm in which an individual salivates at the sight of a visually appealing but unhealthy food. An individual is likely to hold favorable implicit associations toward this food (Raghunathan, Naylor, and Hoyer 2006). Our findings would suggest that as an individual's emotional experiencing ability increases, these associations may become stronger through the individual's nonconscious and automatic desire for hedonic rewards. However, emotional reasoning should ultimately reduce the impact of those associations on subsequent choice through a more deliberative consideration of those associations relative to one's long-term health goals. Because many domains of learning involve the occurrence of affective responses in conjunction with cognitive processing (Baumgartner, Sujan, and Bettman 1992; Krishna and Schwarz 2014), emotional ability effects should extend to various types of learning beyond EC.

It is also important to note how our findings relate to research on affect-as-information and the use of feelings in decision making. Greifeneder, Bless, and Pham (2011) note that feelings are more likely to be used in judgment and decision making when affect is perceived to be related to the item/object being evaluated. To examine whether our model was influenced by affect-as-information processes, we manipulated the perceptual similarity (i.e., relatedness) of USs used in conditioning to the target CS (beer) brands in studies 1 and 2. Our model was robust to differences in perceptual similarity, suggesting that emotional ability affects the transfer of emotions between stimuli regardless of whether affect is perceived to be related to the target of judgment. Thus, emotional ability plays an important role in learned affective responses beyond any effects of affect-as-information processes in judgment and decision making.

Lastly, our research also extends emotional ability research in consumer decision making. Studies of emotional intelligence in marketing research primarily focus on how one's overall emotional ability can improve decision making and choice quality (Kidwell et al. 2008a, 2008b, 2015). To our knowledge, this is the first research to examine the two fundamental areas (experiencing and reasoning) that constitute the higher-order construct of emotional ability within the consumer domain. Furthermore, we also highlight the opposing nature of these areas in their contribution to implicit and explicit attitude formation.

\section{Consumer Implications}

A basic application of EC is to advertising and persuasion. For example, sequential CS-US pairings are more likely to occur in broadcast media, such as a television commercial featuring attractive models that concludes by presenting a brand or product name. Simultaneous CS-US pairings, on the other hand, are more likely in print media, such as a magazine ad that features both a positive US and the brand name on a single page. Depending on differences in emotional ability across various customer segments, we propose that distinct persuasion tactics may be appropriate. Van Rooy, Alonso, and Viswesvaran (2005) highlight a number of group differences in emotional ability, such as gender (women on average have slightly higher emotional ability than men) and age (emotional ability increases with age). As a result, the effectiveness of EC could be directly impacted by these individual differences. For instance, EC should be more effective at changing explicit attitudes among younger consumers as their ability to reason about emotions is likely lower relative to older customer segments.

Furthermore, while EC is unlikely to directly affect explicit attitudes toward mature brands, EC may indirectly influence consumer preferences by enhancing implicit attitudes. This can be particularly powerful in domains where consumers have relatively neutral brand preferences and switching costs are low. However, marketers should take note that segments with enhanced reasoning abilities are less likely to be impacted by EC. For persuasion involving mature brands in these consumer segments, consumers may be more responsive to cognitive appeals or other methods of persuasion beyond associative learning.

\section{Limitations}

While our studies highlight the role of two fundamental areas of emotional ability in shaping EC effects, some limitations should be noted. First, our research does not examine differences in contingency awareness after EC. Awareness of the CS-US pairings post-EC contributes to attitude change (Hofmann et al. 2010), but the measurement of contingency awareness in participants has been subject to debate in the EC literature (Pleyers et al. 2007). Hütter et al. (2012) attempt to address this debate by using the Process Dissociation Procedure (PDP; Jacoby 1998) to identify EC effects across both contingent-aware and -unaware participants. The PDP is superior to traditional contingency awareness techniques, which use recognition tasks, but the PDP cannot generate awareness levels within-subjects and thus was not included here. Future research should examine how contingency awareness 
interacts with emotional ability to impact implicit and explicit attitude change after EC.

It should also be noted that our research measures only emotional ability, albeit with an objective performancebased scoring procedure. However, manipulations that heighten emotional ability should produce similar findings to our research. For example, training emotional ability leads individuals to make food choices that are similar to those who innately possess high levels of emotional ability (Kidwell et al. 2015). Thus, our findings should be robust to whether one's emotional abilities have developed naturally over time or through training programs designed to enhance decision making.

Additionally, our research examines EC only in the domains of beer and soft drinks. Beverages were chosen because food decision making is susceptible to persuasion attempts such as EC (Kerkhof et al. 2009). However, future studies of emotional ability should extend beyond beverages to generalize our findings and further examine our conceptual model.

\section{Future Research}

To our knowledge, the current research provides the first insights into how emotional ability impacts EC effects. However, additional issues warrant consideration in future research. First, researchers should test how emotional ability impacts EC in different sensory modalities beyond the visual displays used in our current studies. For example, Gorn's (1982) seminal study involves the use of music to condition product preference, while other studies have also examined odors in EC effects (Baeyens et al. 1996; Stevenson, Boakes, and Wilson 2000; Todrank et al. 1995). Because sensory modalities such as sound, taste, and smell can elicit strong affective responses, future research should consider if the areas of emotional ability examined here impact EC effects differently across other modalities.

Future research should also examine additional individual differences in information processing and how those interact with emotional ability to impact conditioning effects. Greifeneder et al. (2011) note that individual differences, such as the chronic tendency to focus on one's mood, can enhance the impact of feelings on judgments. Might emotional ability exacerbate or reduce these effects among individuals whose feelings are routinely salient? Additionally, faith in intuition has been shown to moderate the relationship between implicit and explicit CS attitudes (Zimmerman et al. 2011). How might faith in intuition interact with the ability of an individual to reason about emotions when making explicit CS judgments? Furthermore, individuals high in need for cognition exhibit more valenced evaluations after EC (Priluck and Till 2004). The interplay of one's desire for deliberation and ability to reason about emotions should influence how explicit judgments of a CS are formed. We suggest that assessing these and other moderators would provide additional insights into how emotional ability impacts EC effects.

In conclusion, the current research demonstrates the important role that emotional ability plays in EC. Whereas the ability to experience emotions facilitates the creation of implicit associations from EC, the ability to reason about emotions reduces the impact of implicit CS associations on subsequent explicit evaluations. Future research should continue to examine the ways in which emotional ability impacts associative learning processes.

\section{DATA COLLECTION INFORMATION}

The first author collected data for study 1 through the marketing department subject pool at the University of Central Florida in September 2017. The first author collected data for study 2 through the marketing department subject pool at the University of Central Florida in October 2017. The third author collected data for study 3 through Amazon Mechanical Turk in June 2017. The third author collected data for study 4 through the marketing department subject pool at the University of Kentucky in August and September 2017. The first and third authors collected the data for study 5 through Amazon Mechanical Turk in September 2017. All authors reviewed and analyzed data jointly across all studies.

\section{REFERENCES}

Averill, James R. and Elma P. Nunley (1992), Voyages of the Heart: Living an Emotionally Creative Life, New York: Free Press.

Baeyens, Frank, Amy Wrzesniewski, Jan De Houwer, and Paul Eelen (1996), "Toilet Rooms, Body Massages, and Smells: Two Field Studies on Human Evaluative Odor Conditioning," Current Psychology, 15 (1), 77-96.

Balas, Robert and Bertram Gawronski (2012), "On the Intentional Control of Conditioned Evaluative Responses," Learning and Motivation, 43 (3), 89-98.

Baumgartner, Hans, Mita Sujan, and James R. Bettman (1992), "Autobiographical Memories, Affect, and Consumer Information Processing," Journal of Consumer Psychology, 1 (1), 53-82.

Brackett, Marc A., John D. Mayer, and Rebecca M. Warner (2004), "Emotional Intelligence and Its Relation to Everyday Behaviour," Personality and Individual Differences, 36 (6), 1387-402.

Cacioppo, John T., Beverly S. Marshall-Goodell, Louis G. Tassinary, and Richard E. Petty (1992), "Rudimentary Determinants of Attitudes: Classical Conditioning Is More Effective When Prior Knowledge about the Attitude Stimulus Is Low than High," Journal of Experimental Social Psychology, 28 (3), 207-33.

Caruso, David R. and Peter Salovey (2004), The Emotionally Intelligent Manager, San Francisco: Jossey-Bass.

Dedonder, Jonathan, Olivier Corneille, Vincent Yzerbyt, and Toon Kuppens (2010), "Evaluative Conditioning of 
High-Novelty Stimuli Does Not Seem to Be Based on an Automatic Form of Associative Learning," Journal of Experimental Social Psychology, 46 (6), 1118-21.

De Houwer, Jan (2011), "Evaluative Conditioning: A Review of Procedure Knowledge and Mental Process Theories," in Associative Learning and Conditioning Theory: Human and Non-Human Applications, ed. Todd R. Schachtman and Steve Reilly, New York: Oxford University Press, 399-416.

De Houwer, Jan, Sarah Thomas, and Frank Baeyens (2001), "Association Learning of Likes and Dislikes: A Review of 25 Years of Research on Human Evaluative Conditioning," Psychological Bulletin, 127 (6), 853-69.

Dempsey, Melanie A. and Andrew A. Mitchell (2010), "The Influence of Implicit Attitudes on Choice When Consumers Are Confronted with Conflicting Attribute Information," Journal of Consumer Research, 37 (4), 614-25.

Fitness, Julie (2001), "Emotional Intelligence and Intimate Relationships," in Emotional Intelligence in Everyday Life, ed. Joseph Ciarrochi, Hove, UK: Psychology Press, 98-112.

Gawronski, Bertram and Galen V. Bodenhausen (2006), "Associative and Propositional Processes in Evaluation: An Integrative Review of Implicit and Explicit Attitude Change," Psychological Bulletin, 132 (5), 692-731.

Gibson, Bryan (2008), "Can Evaluative Conditioning Change Attitudes toward Mature Brands? New Evidence from the Implicit Association Test," Journal of Consumer Research, 35 (1), 178-88.

Gibson, Bryan, Christopher Redker, and Ian Zimmerman (2014), "Conscious and Nonconscious Effects of Product Placement: Brand Recall and Active Persuasion Knowledge Affect Brand Attitudes and Brand Self-Identification Differently," Psychology of Popular Media Culture, 3 (1), 19-37.

Gorn, Gerald J. (1982), "The Effects of Music in Advertising on Choice Behavior: A Classical Conditioning Approach," Journal of Marketing, 46 (1), 94-101.

Greifeneder, Rainer, Herbert Bless, and Michel Tuan Pham (2011), "When Do People Rely on Affective and Cognitive Feelings in Judgment? A Review," Personality and Social Psychology Review, 15 (2), 107-41.

Gross, James J. (1998), "The Emerging Field of Emotion Regulation: An Integrative Review," Review of General Psychology, 2 (3), 271-99.

Halbeisen, Georg, Katarina Blask, Rebecca Weil, and Eva Walther (2014), "The Role of Recollection in Evaluative Conditioning," Journal of Experimental Social Psychology, 55 (11), 162-8.

Hayes, Andrew F. (2013), Introduction to Mediation, Moderation, and Conditional Process Analysis: A Regression-Based Approach, New York: Guilford.

Hofmann, Wilhelm, Jan De Houwer, Marco Perugini, Frank Baeyens, and Geert Crombez (2010), "Evaluative Conditioning in Humans: A Meta-Analysis," Psychological Bulletin, 136 (3), 390-421.

Hütter, Mandy and Steven Sweldens (2013), "Implicit Misattribution of Evaluative Responses: ContingencyUnaware Evaluative Conditioning Requires Simultaneous Stimulus Presentations," Journal of Experimental Psychology: General, 142 (3), 638-43.

(2018), "Dissociating Controllable and Uncontrollable Effects of Affective Stimuli on Attitudes and Consumption," Journal of Consumer Research, forthcoming.

Hütter, Mandy, Steven Sweldens, Christoph Stahl, Christian Unkelbach, and Karl Christoph Klauer (2012), "Dissociating
Contingency Awareness and Conditioned Attitudes: Evidence of Contingency-Unaware Evaluative Conditioning," Journal of Experimental Psychology: General, 141 (3), 539-57.

Jacoby, Larry L. (1998), "Invariance in Automatic Influences of Memory: Toward a User's Guide for the Process-Dissociation Procedure," Journal of Experimental Psychology: Learning, Memory, and Cognition, 24 (1), 3-26.

Jones, Christopher R., Michael A. Olson, and Russell H. Fazio (2010), "Evaluative Conditioning: The 'How' Question," in Advances in Experimental Social Psychology, Vol. 43, ed. Mark P. Zanna and James M. Olson, Cambridge, MA: Academic Press, 205-55.

Kerkhof, Inneke, Debora Vansteenwegen, Frank Baeyens, and Dirk Hermans (2009), "A Picture-Flavour Paradigm for Studying Complex Conditioning Processes in Food Preference Learning," Appetite, 53 (3), 303-8.

Kidwell, Blair, David M. Hardesty, and Terry L. Childers (2008a), "Consumer Emotional Intelligence: Conceptualization, Measurement, and the Prediction of Consumer Decision Making," Journal of Consumer Research, 35 (1), 154-66.

(2008b), "Emotional Calibration Effects on Consumer Choice," Journal of Consumer Research, 35 (4), 611-21.

Kidwell, Blair, David M. Hardesty, Brian R. Murtha, and Shibin Sheng (2011), "Emotional Intelligence in Marketing Exchanges," Journal of Marketing, 75 (1), 78-95.

Kidwell, Blair, Jonathan Hasford, and David M. Hardesty (2015), "Emotional Ability Training and Mindful Eating," Journal of Marketing Research, 52 (1), 105-19.

Krishna, Aradhna and Norbert Schwarz (2014), "Sensory Marketing, Embodiment, and Grounded Cognition: A Review and Introduction," Journal of Consumer Psychology, 24 (2), 159-68.

Lopes, Paulo N., Marc A. Brackett, John B. Nezlek, Astrid Schütz, Ina Sellin, and Peter Salovey (2004), "Emotional Intelligence and Social Interaction," Personality and Social Psychology Bulletin, 30 (8), 1018-34.

Martins, Alexandra, Nelson Ramalho, and Estelle Morin (2010), "A Comprehensive Meta-Analysis of the Relationship between Emotional Intelligence and Health," Personality and Individual Differences, 49 (6), 554-64.

Mayer, John D. and Peter Salovey (1997), "What Is Emotional Intelligence?" in Emotional Development and Emotional Intelligence: Implications for Educators, ed. Peter Salovey and David Slusher, New York: Basic Books, 3-31.

Mayer, John D., Peter Salovey, and David Caruso (2000), "Models of Emotional Intelligence," in The Handbook of Intelligence, ed. Robert J. Sternberg, New York: Cambridge University Press, 396-420.

Montoya, Amanda K. (2017), email message to authors, June $12^{\text {th }}$, 2017.

Montoya, Amanda K. and Andrew F. Hayes (2017), "Two-Condition Within-Participant Statistical Mediation Analysis: A PathAnalytic Framework," Psychological Methods, 22 (1), 6-27.

Olson, Michael A. and Russell H. Fazio (2001), "Implicit Attitude Formation through Classical Conditioning," Psychological Science, 12 (5), 413-7.

Ortony, Andrew, Gerald L. Clore, and Allan Collins (1988), The Cognitive Structure of Emotions, Cambridge, UK: Cambridge University Press.

Payne, B. Keith, Clara Michelle Cheng, Olesya Govorun, and Brandon D. Stewart (2005), “An Inkblot for Attitudes: Affect 
Misattribution as Implicit Measurement," Journal of Personality and Social Psychology, 89 (3), 277-93.

Pham, Michel Tuan (1998), "Representativeness, Relevance, and the Use of Feelings in Decision Making," Journal of Consumer Research, 25 (2), 144-59.

Pleyers, Gordy, Olivier Corneille, Olivier Luminet, and Vincent Yzerbyt (2007), "Aware and (Dis)Liking: Item-Based Analyses Reveal That Valence Acquisition via Evaluative Conditioning Emerges Only When There Is Contingency Awareness," Journal of Experimental Psychology: Learning, Memory, and Cognition, 33 (1), 130-44.

Priluck, Randi and Brian D. Till (2004), "The Role of Contingency Awareness, Involvement, and Need for Cognition in Attitude Formation," Journal of the Academy of Marketing Science, 32 (3), 329-44.

Raghunathan, Rajagopal, Rebecca Walker Naylor, and Wayne D. Hoyer (2006), "The Unhealthy = Tasty Intuition and Its Effects on Taste Inferences, Enjoyment, and Choice of Food Products," Journal of Marketing, 70 (4), 170-84.

Roseman, Ira J. (1984), "Cognitive Determinants of Emotions: A Structural Theory," in Review of Personality and Social Psychology: Vol. 5. Emotions, Relationships, and Health, ed. P. Shaver, Beverly Hills, CA: Sage, 11-36.

Shimp, Terence A., Elnora W. Stuart, and Randall W. Engle (1991), “A Program of Classical Conditioning Experiments Testing Variations in the Conditioned Stimulus and Context," Journal of Consumer Research, 18 (1), 1-12.

Stevenson, Richard J., Robert A. Boakes, and Judith P. Wilson (2000), "Resistance to Extinction of Conditioned Odor Perceptions: Evaluative Conditioning Is Not Unique," Journal of Experimental Psychology: Learning, Memory, and Cognition, 26 (2), 423-40.
Sweldens, Steven, Olivier Corneille, and Vincent Yzerbyt (2014), "The Role of Awareness in Attitude Formation through Evaluative Conditioning," Personality and Social Psychology Review, 18 (2), 187-209.

Sweldens, Steven, Stijn M. J. Van Osselaer, and Chris Janiszewski (2010), "Evaluative Conditioning Procedures and the Resilience of Conditioned Brand Attitudes," Journal of Consumer Research, 37 (3), 473-89.

Todrank, Josephine, Deidre Byrnes, Amy Wrzesniewski, and Paul Rozin (1995), "Odors Can Change Preferences for People in Photographs: A Cross-Modal Evaluative Conditioning Study with Olfactory USs and Visual CSs," Learning and Motivation, 26 (2), 116-40.

Van Rooy, David L., Alexander Alonso, and Chockalingam Viswesvaran (2005), "Group Differences in Emotional Intelligence Scores: Theoretical and Practical Implications," Personality and Individual Differences, 38 (3), 689-700.

Yim, Chi Kin, David K. Tse, and Kimmy Wa Chan (2008), "Strengthening Customer Loyalty through Intimacy and Passion: Roles of Customer-Firm Affection and CustomerStaff Relationships in Services," Journal of Marketing Research, 45 (6), 741-56.

Zanon, Riccardo, Jan De Houwer, Anne Gast, and Colin Tucker Smith (2014), "When Does Relational Information Influence Evaluative Conditioning?" Quarterly Journal of Experimental Psychology, 67 (11), 2105-22.

Zimmerman, Ian, Christopher Redker, and Bryan Gibson (2011), "The Role of Faith in Intuition, Need for Cognition and Method of Attitude Formation in Implicit-Explicit Brand Attitude Relationship Strength," Journal of Consumer Psychology, 21 (3), 290-301. 